\title{
Genotypic distribution of multidrug-resistant and extensively drug-resistant tuberculosis in northern Thailand
}

\author{
Risara Jaksuwan' \\ Prasit Tharavichikul ${ }^{2}$ \\ Jayanton Patumanond ${ }^{3}$ \\ Charoen Chuchottaworn ${ }^{4}$ \\ Sakarin Chanwong ${ }^{5}$ \\ Saijai Smithtikarn ${ }^{6}$ \\ Jongkolnee Settakorn ${ }^{7}$ \\ 'Clinical Epidemiology Unit, \\ ${ }^{2}$ Department of Microbiology, Faculty \\ of Medicine, Chiang Mai University, \\ Chiang Mai, ${ }^{3}$ Division of Clinical \\ Epidemiology, Faculty of Medicine, \\ Thammasat University, Pathum Thani, \\ ${ }^{4}$ Division of Respiratory Medicine, \\ Chest Disease Institute, Nonthaburi, \\ ${ }^{5}$ Office of Disease Prevention and \\ Control Region I0, Chiang Mai, \\ ${ }^{6}$ Bureau of Tuberculosis, Department \\ of Disease Control, Ministry of Public \\ Health, Bangkok, ${ }^{7}$ Department of \\ Pathology, Faculty of Medicine, Chiang \\ Mai University, Chiang Mai, Thailand
}

Correspondence: Jongkolnee Settakorn Department of Pathology, Faculty of Medicine, Chiang Mai University, Inthavaroros Road, Sriphum sub-district, Mueang Chiang Mai District, Chiang Mai Province 50200, Thailand

Tel +66 $53945442-4$

Fax +66 53217144

Email jsettakorn@gmail.com
This article was published in the following Dove Press journal:

Infection and Drug Resistance

10 June 2017

Number of times this article has been viewed

Background: Multidrug/extensively drug-resistant tuberculosis (M/XDR-TB) is a major public health problem, and early detection is important for preventing its spread. This study aimed to demonstrate the distribution of genetic site mutation associated with drug resistance in $\mathrm{M} / \mathrm{XDR}-\mathrm{TB}$ in the northern Thai population.

Methods: Thirty-four clinical MTB isolates from M/XDR-TB patients in the upper northern region of Thailand, who had been identified for drug susceptibility using the indirect agar proportion method from 2005 to 2012, were examined for genetic site mutations of katG, inhA, and $a h p C$ for isoniazid (INH) drug resistance and $r p o B$ for rifampicin (RIF) drug resistance. The variables included the baseline characteristics of the resistant gene, genetic site mutations, and drug susceptibility test results.

Results: All 34 isolates resisted both INH and RIF. Thirty-two isolates (94.1\%) showed a mutation of at least 1 codon for $k a t G, i n h A$, and $a h p C$ genes. Twenty-eight isolates $(82.4 \%)$ had a mutation of at least 1 codon of $r p o B$ gene. The katG, inhA, ahpC, and $r p o B$ mutations were detected in 20 (58.7\%), 27 (79.4\%), $13(38.2 \%)$, and $28(82.3 \%)$ of 34 isolates. The 3 most common mutation codons were katG 315 (11/34, 35.3\%), inhA 14 (11/34, 32.4\%), and inhA $114(11 / 34,32.4 \%)$. For this population, the best genetic mutation test panels for INH resistance included 8 codons ( $k a t G 310$, katG 340, katG 343, inhA 14, inhA 84, inhA 86, inhA 114, and $a h p C 75)$, and for RIF resistance included 6 codons ( $r p o B$ 445, rpoB 450, rрoB 464, rpoB 490, $r p o B 507$, and $r p o B 508$ ) with a sensitivity of $94.1 \%$ and $82.4 \%$, respectively.

Conclusion: The genetic mutation sites for drug resistance in M/XDR-TB are quite variable. The distribution of these mutations in a certain population must be studied before developing the specific mutation test panels for each area. The results of this study can be applied for further molecular M/XDR-TB diagnosis in the upper northern region of Thailand.

Keywords: tuberculosis, drug resistance, MDR-TB, XDR-TB, genotype, mutation

\section{Introduction}

Tuberculosis (TB) is an important global problem, especially multidrug-resistant TB (MDR-TB) and extensively drug-resistant TB (XDR-TB). In 2015, the world-wide estimation of MDR-TB cases and deaths was 80,000 and 250,000, respectively. ${ }^{1}$ MDR-TB is caused by Mycobacterium tuberculosis (MTB), which resists at least 2 drugs (isoniazid [INH] and rifampicin [RIF]). Pre-XDR-TB is a disease caused by the M. tuberculosis strain, which resists INH and RIF and either a fluoroquinolone or a second-line injectable drug, but not both. ${ }^{1}$ XDR-TB is defined as MDR-TB, which adds more resistance to any fluoroquinolone and at least 1 of 3 injectable second-line drugs (amikacin, capreomycin, and kanamycin [KAN]). ${ }^{2}$ Approximately $9.5 \%$ of MDR-TB 
patients developed XDR-TB. ${ }^{1}$ Unfortunately, about $20 \%$ of MDR-TB cases were undetectable. ${ }^{1}$ Thailand is among the countries with a TB burden, as it was ranked within the top 20 countries with TB in $2015 .{ }^{1}$

Early and accurate detection of M/XDR-TB is important for effectively treating and preventing its transmission. ${ }^{3-6}$ However, the phenotypic drug susceptibility test (DST) takes weeks or months for culturing MTB and identifying the resisted drugs and requires a high level of microbiological safety. ${ }^{7,8}$ Diagnosis and treatment delays are among the major causes of the spread of the disease, which interferes with the TB control programs. A rapid molecular TB-DST test greatly increases the efficacy of MDR-TB treatment ${ }^{5,6,9}$ as the minimal turnaround time is $<1$ day. ${ }^{10,11}$

As the distribution of mutation in drug-resistant genes is region specific, knowing the epidemiology of the genetic drug resistance of M/XDR-TB in certain areas would be beneficial for developing specific and rapid molecular tests, ${ }^{8,12}$ as well as treatment protocols, ${ }^{13}$ because the prevalence of such resistance differs in various populations. ${ }^{14-16}$ Many studies have focused on genetic drug resistance in MTB. INH is the main drug used; it is an effective antiTB drug and has been used till date. ${ }^{2,11}$ The mechanism of INH resistance in MTB is associated with the mutations of $k a t G$, inh $A$, and $a h p C .{ }^{17-19}$ The $k a t G$ mutation was found in high levels of INH resistance, ${ }^{20,21}$ and the inhA mutation was found at low levels or monoresistance to INH. ${ }^{20,22} \mathrm{RIF}$ is combined with INH as the main anti-TB drug, and it is inferred with transcription by the DNA-dependent RNA polymerase by binding to the $\beta$-subunit hindering transcription organisms related to rpoB 531 mutation, ${ }^{23-25}$ which destroys the organisms. ${ }^{26,27}$

In this study, we examined the distribution of drugresistant gene mutations, including $k a t G$, inh $A, \operatorname{ahp} C$, and $r p o B$, in $\mathrm{M} / \mathrm{XDR}-\mathrm{TB}$ isolates in the northern Thai population. Then, we identified the best mutation test panels for INH and RIF resistance.

\section{Materials and methods}

Two hundred and sixty-one M/XDR-TB isolates first diagnosed with MDR-TB or XDR-TB from patients between January 2005 and June 2012 were retrieved from an archive of the Laboratory of the Office of Disease Prevention and Control Region 10 (DPC10). According to the World Health Organization (WHO) criteria, MDR isolates resisted at least INH and RIF; and XDR isolates resisted INH, RIF, ofloxacin (OFX), and KAN. DPC10 hosts a regional TB laboratory, covering 8 provinces in the north of Thailand, and the isolates were subcultured. Only 34 specimens $(9.5 \%)$ were grown in $5 \mathrm{~mL}$ of $7 \mathrm{H} 9$ broth supplemented with PANTA in 6 weeks and 3\% Ogawa within 8 weeks. The bacterial agents were further examined for phenotypic DST and DNA sequencing.

\section{Phenotypic drug susceptibility test}

Thirty-four growing M/XDR-TB isolates were tested for first- and second-line drug resistance (INH, RIF, OFX, and KAN) using the proportion method with Lowensentein Jensen (LJ) medium ${ }^{28}$ at DPC10 laboratory. The DOTS-plus DST was performed according to the WHO guidelines, ${ }^{29}$ and all suspected TB and MDR-TB patients were investigated using 3 methods: 3 direct acid-fast bacilli sputum smear examinations, a Mycobacterium culture, and first-line DST ${ }^{29}$ during the first diagnosis. Additionally, at the same time, a molecular test was carried out in order to confirm M. tuberculosis. DST was determined using the indirect agar proportion method with INH, RIF, streptomycin, and ethambutol. If there was resistance to INH and/or RIF, a second-line anti-TB DST was performed with OFX and KAN. For our study, LJ medium was supplemented individually with anti-TB drugs. INH $(0.2 \mu \mathrm{g} / \mathrm{mL}), \operatorname{RIF}(40.0 \mu \mathrm{g} / \mathrm{mL})$, OFX $(2.0 \mu \mathrm{g} / \mathrm{mL})$, and $\mathrm{KAN}(30 \mu \mathrm{g} / \mathrm{mL})$ were repeated in order to confirm that the results were the same as the previous results; if the results were not the same then they were excluded from the study.

\section{DNA extraction}

Thirty-four M/XDR-TB isolates were cultured on solid media (LJ and OGAWA). Chromosomal DNA was extracted using the MolecuTech REBA MTB-MDR 2011 commercial kit. Briefly, 1 loop ( $0.2 \mu \mathrm{L}$ diameter sterile inoculating loop) of the cultured bacteria was resuspended in $50 \mu \mathrm{L}$ of DNA extraction solution. The solution was vortexed for $1 \mathrm{~min}$, boiled for $10 \mathrm{~min}$, and centrifuged $(13,000 \mathrm{rpm})$ for $3 \mathrm{~min}$ at room temperature. Then, $5-10 \mu \mathrm{L}$ of the supernatant was transferred to a microtube (cryovial tube). The purified DNA pellet was stored at $4^{\circ} \mathrm{C}$.

\section{Sequencing method}

The polymerase chain reaction (PCR) and DNA sequencing parts were carried out at Macrogen Laboratory, Seoul, Republic of Korea. Four genetic loci, $k a t G$, inhA, ahpC (INH), and rpoB (RIF), were amplified by PCR, as shown in Table S1 ${ }^{30,31}$ The used primers are shown in Table S1. The amplified products were purified with a multiscreen filter plate (Millipore Corp., Bedford, MA, USA). 
Table I Frequency of genetic site mutations of katG, inhA, ahpC, and rpoB genes in $34 \mathrm{M} / \mathrm{XDR}$-TB isolates

\begin{tabular}{|c|c|c|c|c|c|c|c|}
\hline katG & n (\%) & inhA & n (\%) & $a h p C$ & n (\%) & rpoB & n (\%) \\
\hline katG 229 & I (2.9) & $\operatorname{inh} A 14$ & II (32.4) & $\operatorname{ahpC} 10$ & 4 (II.8) & $r p o B 445$ & $8(23.5)$ \\
\hline katG 300 & I (2.9) & $\operatorname{inh} A 25$ & I (2.9) & $\operatorname{ahpC~} 12$ & $4(11.8)$ & rров 450 & $6(17.7)$ \\
\hline katG 302 & I (2.9) & inhA 78 & $8(23.5)$ & $a h p C 20$ & $4(I \mid .8)$ & rpoB 464 & $6(17.7)$ \\
\hline katG 308 & $3(8.8)$ & $\operatorname{inh} A 81$ & $7(20.6)$ & $\operatorname{ahpC} 22$ & $3(8.8)$ & rров 483 & $4(I 1.8)$ \\
\hline katG 310 & $3(8.8)$ & inhA 84 & $9(26.5)$ & $a h p C 75$ & 7 (20.6) & rрoв 490 & $7(20.6)$ \\
\hline katG 312 & $4(I I .8)$ & inhA 86 & $8(23.5)$ & $a h p C 76$ & $6(17.7)$ & rров 493 & $4(11.8)$ \\
\hline katG 3।4 & I (2.9) & $\operatorname{inh} A 94$ & $3(8.8)$ & & & rpoB 507 & $9(26.5)$ \\
\hline katG 315 & $12(35.3)$ & $\operatorname{inh} A|| 4$ & II (32.4) & & & rpoB 508 & $9(26.5)$ \\
\hline katG 320 & I (2.9) & & & & & & \\
\hline katG 340 & 4 (II.8) & & & & & & \\
\hline katG 343 & $7(20.6)$ & & & & & & \\
\hline
\end{tabular}

Abbreviation: $M / X D R-T B$, multidrug/extensively drug-resistant tuberculosis.

\section{Analysis \\ DNA sequencing analysis}

The sequencing data obtained from the ABI3730XL DNA analyzer were investigated for the presence or absence of mutations by alignments with the corresponding nucleotide sequences of $M$. tuberculosis (H37RV) using the NCBI nucleotide blast program.

\section{Data analysis}

The phenotypic DST and genetic site mutation for the DST data sets were compiled using an Excel 2010 database. Statistical analysis was performed using STATA version 11.0. The resistant genes and genetic site mutations were presented according to frequency and percentage.

\section{Results}

Among the 34 growing M/XDR-TB isolates, 24 (70.5\%) MDR-TB, 9 (26.5\%) Pre-XDR-TB, and 1 (3.0\%) XDR-TB were identified. All of the isolates were resistant to both INH and RIF. The frequency and patterns of genetic loci mutation are demonstrated in Tables 1 and 2. Table 3 exhibits the raw data of genetic mutation in all 34 isolates. Mutations were found, including $k a t G$ in 20 isolates (58.8\%), inhA in 27 isolates (79.4\%), ahpC in 13 isolates (38.2\%), and $r p o B$ in 28 isolates $(82.3 \%)$. INH-resistant gene mutations were found in 32 isolates ( $94.1 \%$ sensitivity). RIF-resistant gene mutations were identified in 28 isolates ( $82.4 \%$ sensitivity).

One isolate revealed no mutation for the studied genetic loci (no. 18). Two isolates showed no mutation for katG, inhA, or $a h p C$. (no. 2 and 18). For the INH-resistant genes, a single-gene mutation was found in 10 isolates (29.4\%), 2 for $k a t G, 7$ for $i n h A$, and 1 for $a h p C$. Double mutation was found in 16 isolates (47.1\%), 10 for katG/inhA, 4 for ihnA/ $a h p C$, and 2 for katG/ahpC. Triple katG/inhA/ahpC mutation was seen in 6 isolates (17.6\%). Regarding the RIF-resistant
Table 2 Distribution of Mycobacterium tuberculosis genetic mutations with isoniazid and rifampicin resistance

\begin{tabular}{|c|c|c|}
\hline Gene mutation codon & $\mathbf{n}$ & $\%$ \\
\hline \multicolumn{3}{|l|}{ katG } \\
\hline No mutation & 14 & 41.3 \\
\hline katG 315 & 9 & 26.7 \\
\hline katG 320 & I & 2.9 \\
\hline katG 312 & I & 2.9 \\
\hline katG 343 & I & 2.9 \\
\hline$k a t G 315$ and $k a t G 343$ & I & 2.9 \\
\hline katG 308 and $k a t G 310$ & I & 2.9 \\
\hline katG 312 and $k a t G 343$ & 2 & 5.9 \\
\hline katG 308, katG 315, and katG 340 & I & 2.9 \\
\hline katG 3I5, katG 340, and katG 343 & I & 2.9 \\
\hline katG 310, katG 314, katG 340, and katG 343 & I & 2.9 \\
\hline katG 299, katG 300, katG 302, katG 310, and katG 312 & I & 2.9 \\
\hline \multicolumn{3}{|l|}{$\operatorname{Inh} A$} \\
\hline No mutation & 7 & 20.6 \\
\hline $\operatorname{inh} A$ I4 & 6 & 17.7 \\
\hline $\operatorname{inh} A 25$ & I & 2.9 \\
\hline $\operatorname{inh} A 86$ & I & 2.9 \\
\hline $\operatorname{inh} A \mid 14$ & 5 & 14.8 \\
\hline inhA 14 and inhA II4 & 4 & 11.8 \\
\hline inhA 84 and inhA II 4 & I & 2.9 \\
\hline $\operatorname{inh} A$ 78, inhA 8I, and inhA 84 & I & 2.9 \\
\hline $\operatorname{inh} A$ 78, inhA 8I, and inhA 86 & I & 2.9 \\
\hline $\operatorname{inh} A$ 78, inhA 84, and inhA 86 & I & 2.9 \\
\hline inhA I4, inhA 78, inhA 8I, and inhA 84 & I & 2.9 \\
\hline inhA 78, inhA 8I, inhA 84, and inhA 86 & I & 2.9 \\
\hline $\operatorname{inh} A$ 78, inhA 84, inhA 86, and inhA II4 & I & 2.9 \\
\hline $\operatorname{inh} A 81, \operatorname{inh} A$ 84, inhA 86, and inhA 94 & I & 2.9 \\
\hline inhA 78, inhA 8I, inhA 84, inhA 86, and inhA 94 & 2 & 5.9 \\
\hline \multicolumn{3}{|l|}{$\operatorname{ahpC}$} \\
\hline No mutation & 21 & 61.8 \\
\hline $\operatorname{ahpC} 10$ & 1 & 2.9 \\
\hline $\operatorname{ahpC~} 75$ & I & 2.9 \\
\hline$a h p C 10$ and $a h p C 12$ & I & 2.9 \\
\hline$a h p C 12$ and $a h p C 20$ & I & 2.9 \\
\hline$a h p C 75$ and $a h p C 76$ & 6 & 17.7 \\
\hline$a h p C 10, a h p C 20$, and $a h p C 22$ & I & 2.9 \\
\hline$a h p C 12, a h p C 20$, and $a h p C 22$ & I & 2.9 \\
\hline$a h p C 10, a h p C 12, a h p C 20$, and $a h p C 22$ & I & 2.9 \\
\hline
\end{tabular}


Table 2 (Continued)

\begin{tabular}{|c|c|c|}
\hline Gene mutation codon & $\mathbf{n}$ & $\%$ \\
\hline \multicolumn{3}{|l|}{$r p o B$} \\
\hline No mutation & 6 & 17.7 \\
\hline rров 445 & 2 & 5.9 \\
\hline rpoB 508 & I & 2.9 \\
\hline rpoB 464 & 2 & 5.9 \\
\hline rpoB 450 & 3 & 8.8 \\
\hline rрoв 490 & 2 & 5.9 \\
\hline rpoB 507 & 3 & 8.8 \\
\hline rpoB 445 and rpoB 508 & I & 2.9 \\
\hline rpoB 507 and rpoB 508 & 6 & 17.7 \\
\hline rpoB 445 and rpoB 483 & 1 & 2.9 \\
\hline rpoB 464 and rpoB 483 & 1 & 2.9 \\
\hline rрoB 445, rрoB 464, and rpoB 504 & 1 & 2.9 \\
\hline rров 445, гров 490, and rров 493 & I & 2.9 \\
\hline rрoB 450, rров 490, and rpoB 493 & I & 2.9 \\
\hline rров 445, гров 464, гров 490, and rров 493 & I & 2.9 \\
\hline rров 450, гров 490, гров 483, and rров 493 & 1 & 2.9 \\
\hline rров 445, гров 464, гров 490, гров 483, and rров 493 & I & 2.9 \\
\hline
\end{tabular}

gene, no rроB mutation was found in 6 isolates (no. 5, 8, $11,15,8$, and 34).

The common mutated genetic loci found in M/XDR-TB were katG $315(\mathrm{n}=12,35.3 \%)$, inhA $14(\mathrm{n}=11,32.4 \%)$, ihnA $114(\mathrm{n}=11,32.4 \%), \operatorname{ihnA} 84(\mathrm{n}=9,26.5 \%)$, rрoB $507(\mathrm{n}=9$, $26.47 \%)$, and rpoB $508(\mathrm{n}=9,26.47 \%)$.

\section{Discussion}

The frequency and distribution of drug-resistant mutations are variable across regions and countries (Table 4). The frequency of kat $G$ mutation ranged from $58.5 \%$ to $93.7 \%$, ${ }^{39}$ and the most commonly mutated katG locus was katG $315 .{ }^{32-37,39,40}$ The reported frequency of inhA mutation was $4.7 \%-79.4 \%,{ }^{32,33}$ and the most commonly mutated inh $A$ locus was inh $A 15 .^{32-40}$ The frequency of $\operatorname{ahpC}$ mutation was relatively low $(9.1 \%-38.2 \%)^{34,35}$ and was extremely

Table 3 Raw distribution of genetic site mutations of $k a t G$, inhA, ahpC, and rpoB genes in M/XDR-TB isolates

\begin{tabular}{|c|c|c|c|c|c|c|c|c|c|c|c|c|c|c|c|c|}
\hline No. & $\begin{array}{l}\text { katG } \\
299\end{array}$ & $\begin{array}{l}\text { katG } \\
300\end{array}$ & $\begin{array}{l}\text { katG } \\
302\end{array}$ & $\begin{array}{l}\text { katG } \\
308\end{array}$ & $\begin{array}{l}\text { katG } \\
310\end{array}$ & $\begin{array}{l}\text { katG } \\
312\end{array}$ & $\begin{array}{l}\text { katG } \\
314\end{array}$ & $\begin{array}{l}\text { katG } \\
315\end{array}$ & $\begin{array}{l}\text { katG } \\
320\end{array}$ & $\begin{array}{l}\text { katG } \\
340\end{array}$ & $\begin{array}{l}\text { katG } \\
343\end{array}$ & $\begin{array}{l}\text { inhA } \\
14\end{array}$ & $\begin{array}{l}\operatorname{inh} A \\
25\end{array}$ & $\begin{array}{l}\text { inhA } \\
78\end{array}$ & $\begin{array}{l}\text { inhA } \\
81\end{array}$ & $\begin{array}{l}\text { inhA } \\
84\end{array}$ \\
\hline 1 & $Y$ & $Y$ & $Y$ & $Y$ & $Y$ & $Y$ & - & - & - & - & - & - & - & - & - & - \\
\hline 2 & - & - & - & - & - & - & - & - & - & - & - & - & - & - & - & - \\
\hline 3 & - & - & - & - & - & - & - & - & - & - & $Y$ & - & $Y$ & - & - & - \\
\hline 4 & - & - & - & - & - & $Y$ & - & - & - & - & $Y$ & - & - & - & - & $Y$ \\
\hline 5 & - & - & - & $Y$ & $Y$ & - & - & - & - & - & - & - & - & - & - & - \\
\hline 6 & - & - & - & - & - & - & - & $Y$ & - & - & - & $Y$ & - & - & - & - \\
\hline 7 & - & - & - & - & - & - & - & $Y$ & - & $Y$ & $Y$ & $Y$ & - & - & - & - \\
\hline 8 & - & - & - & - & - & - & - & - & - & - & - & $Y$ & - & - & - & - \\
\hline 9 & - & - & - & - & - & - & - & $Y$ & - & - & $Y$ & $Y$ & - & - & - & - \\
\hline 10 & - & - & - & - & - & $Y$ & - & - & - & - & $Y$ & - & - & $Y$ & $Y$ & $Y$ \\
\hline II & - & - & - & - & - & - & - & - & - & - & - & - & - & - & - & - \\
\hline 12 & - & - & - & $Y$ & - & - & - & $Y$ & - & $Y$ & - & $Y$ & - & - & - & - \\
\hline 13 & - & - & - & - & - & - & - & - & - & - & - & - & - & - & - & - \\
\hline 14 & - & - & - & - & - & - & - & $Y$ & - & - & - & - & - & - & - & - \\
\hline 15 & - & - & - & - & - & - & - & - & - & - & - & $Y$ & - & - & - & - \\
\hline 16 & - & - & - & - & - & - & - & - & - & - & - & - & - & - & - & - \\
\hline 17 & - & - & - & - & - & $Y$ & - & - & - & $Y$ & $Y$ & - & - & $Y$ & - & $Y$ \\
\hline 18 & - & - & - & - & - & - & - & - & - & - & - & - & - & - & - & - \\
\hline 19 & - & - & - & - & - & - & - & - & - & - & - & $Y$ & - & - & - & - \\
\hline 20 & - & - & - & - & - & - & - & - & - & - & - & - & - & $Y$ & - & $Y$ \\
\hline 21 & - & - & - & - & - & - & - & - & - & - & - & $Y$ & - & $Y$ & $Y$ & $Y$ \\
\hline 22 & - & - & - & - & - & - & - & $Y$ & - & - & - & - & - & - & - & - \\
\hline 23 & - & - & - & - & $Y$ & - & $Y$ & - & - & $Y$ & $Y$ & - & - & $Y$ & $Y$ & $Y$ \\
\hline 24 & - & - & - & - & - & - & - & $Y$ & - & - & - & - & - & $Y$ & $Y$ & - \\
\hline 25 & - & - & - & - & - & - & - & $Y$ & - & - & - & $Y$ & - & - & - & - \\
\hline 26 & - & - & - & - & - & - & - & - & - & - & - & $Y$ & - & - & - & - \\
\hline 27 & - & - & - & - & - & - & - & - & - & - & - & $Y$ & - & - & - & - \\
\hline 28 & - & - & - & - & - & - & - & $Y$ & - & - & - & - & - & $Y$ & $Y$ & $Y$ \\
\hline 29 & - & - & - & - & - & - & - & - & - & - & - & - & - & $Y$ & $Y$ & $Y$ \\
\hline 30 & - & - & - & - & - & - & - & - & $Y$ & - & - & - & - & - & $Y$ & $Y$ \\
\hline 31 & - & - & - & - & - & - & - & $Y$ & - & - & - & - & - & - & - & - \\
\hline 32 & - & - & - & - & - & - & - & $Y$ & - & - & - & - & - & - & - & - \\
\hline 33 & - & - & - & - & - & - & - & - & - & - & - & - & - & - & - & - \\
\hline 34 & - & - & - & - & - & - & - & $Y$ & - & - & - & - & - & - & - & - \\
\hline
\end{tabular}

Notes: -, no mutation; $Y$, presence of mutation; gray column, proposed mutation test panels.

Abbreviation: M/XDR-TB, multidrug/extensively drug-resistant tuberculosis. 
variable. The frequency of $r p o B$ mutation was relatively high $(80.8 \%-97.0 \%),{ }^{33,34,39}$ predominantly at the locus rpoB 531.

Genomic tests or multiple genetic loci tests are not suitable for resource-limited countries. Developing test panels for M/ XDR-TB drug-resistant genes would be more cost-effective if the test panels of the selected genetic loci provide high sensitivity and specificity. However, as the distributions of the gene mutations across the regions varied, a deeper analysis should be considered in further studies. The distribution of mutation patterns should be examined in each geographical areas before routine diagnostic services being implemented. According to the results of this study, we selected $8 \mathrm{INH}$-resistant gene mutation loci (katG 310, katG 340, katG 343, inhA 14, inhA

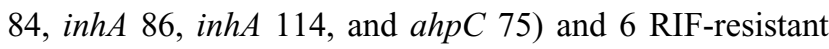
gene mutation loci (rpoB 445, rроB 450, rрoB 464, rрoB $490, r p o B 507$, and $r p o B$ 508) as the best panels for genetic drug resistance tests. Both the panels were able to detect all isolates with at least 1 mutation site for any katG/inhA/ahpC mutation and rpoB mutation. The sensitivities of the proposed INH resistance mutation test was $94.1 \%(32 / 34)$, while that of the RIF resistance mutation test was $82.4 \%(28 / 34)$.

\section{Limitations}

The limitations of this study were the small sample size and the retrospective design. Only 34 isolates were available from a total of 261 isolates. Another weak point was that there was no control group, such as non-M/XDR-TB isolates.

\section{Conclusion}

The results of this study confirm the presence of genetic drug-resistant mutations in the M/XDR-TB isolates in upper northern Thailand. We showed that our mutation patterns

\begin{tabular}{|c|c|c|c|c|c|c|c|c|c|c|c|c|c|c|c|c|}
\hline $\begin{array}{l}\text { inhA } \\
86\end{array}$ & $\begin{array}{l}\text { inhA } \\
94\end{array}$ & $\begin{array}{l}\text { inhA } \\
114\end{array}$ & $\begin{array}{l}\text { ahpC } \\
10\end{array}$ & $\begin{array}{l}a h p C \\
12\end{array}$ & $\begin{array}{l}a h p C \\
20\end{array}$ & $\begin{array}{l}a h p C \\
22\end{array}$ & $\begin{array}{l}\operatorname{ahpC} C \\
75\end{array}$ & $\begin{array}{l}a h p C \\
76\end{array}$ & $\begin{array}{l}r p \circ B \\
445\end{array}$ & $\begin{array}{l}\text { rpoB } \\
450\end{array}$ & $\begin{array}{l}r p \circ B \\
464\end{array}$ & $\begin{array}{l}r p \circ B \\
483\end{array}$ & $\begin{array}{l}\text { rpoB } \\
490\end{array}$ & $\begin{array}{l}r p \circ B \\
493\end{array}$ & $\begin{array}{l}r p \circ B \\
507\end{array}$ & $\begin{array}{l}\text { rpoB } \\
508\end{array}$ \\
\hline - & - & - & - & - & - & - & - & - & $Y$ & - & - & - & - & - & - & $Y$ \\
\hline - & - & - & - & - & - & - & - & - & $Y$ & - & $Y$ & - & - & - & - & $Y$ \\
\hline - & - & - & $Y$ & - & - & - & - & - & - & - & - & - & $Y$ & - & - & - \\
\hline - & - & $Y$ & - & - & - & - & - & - & - & - & - & - & - & - & $Y$ & $Y$ \\
\hline - & - & - & - & - & - & - & - & - & - & - & - & - & - & - & - & - \\
\hline - & - & $Y$ & - & - & - & - & - & - & $Y$ & - & - & $Y$ & - & - & - & - \\
\hline - & - & $Y$ & - & $Y$ & $Y$ & $Y$ & - & - & $Y$ & - & $Y$ & $Y$ & $Y$ & $Y$ & - & - \\
\hline - & - & $Y$ & $Y$ & $Y$ & $Y$ & $Y$ & - & - & - & - & - & - & - & - & - & - \\
\hline - & - & - & - & - & - & - & - & - & - & $Y$ & - & - & $Y$ & $Y$ & - & - \\
\hline$Y$ & - & - & - & - & - & - & - & - & $Y$ & - & - & - & $Y$ & $Y$ & - & - \\
\hline - & - & - & - & - & - & - & $Y$ & $Y$ & - & - & - & - & - & - & - & - \\
\hline - & - & - & $Y$ & - & $Y$ & $Y$ & - & - & - & - & - & - & - & - & $Y$ & $Y$ \\
\hline - & - & $Y$ & - & - & - & - & - & - & - & - & - & - & - & - & $Y$ & - \\
\hline - & - & $Y$ & $Y$ & $Y$ & - & - & - & - & $Y$ & - & - & - & - & - & - & - \\
\hline - & - & - & - & - & - & - & $Y$ & $Y$ & - & - & - & - & - & - & - & - \\
\hline$Y$ & - & - & - & - & - & - & - & - & - & - & - & - & $Y$ & - & - & - \\
\hline$Y$ & - & $Y$ & - & - & - & - & - & - & - & $Y$ & - & $Y$ & $Y$ & $Y$ & - & - \\
\hline - & - & - & - & - & - & - & - & - & - & - & - & - & - & - & - & - \\
\hline - & - & - & - & - & - & - & - & - & - & $Y$ & - & - & - & - & - & - \\
\hline$Y$ & - & - & - & - & - & - & $Y$ & - & $Y$ & $Y$ & $Y$ & - & $Y$ & - & - & - \\
\hline - & - & - & - & - & - & - & - & - & $Y$ & - & - & - & - & - & - & - \\
\hline - & - & $Y$ & - & - & - & - & - & - & - & $Y$ & - & - & - & - & - & - \\
\hline$Y$ & $Y$ & - & - & $Y$ & $Y$ & - & - & - & - & $Y$ & - & - & - & - & - & - \\
\hline$Y$ & - & - & - & - & - & - & - & - & - & - & - & - & - & - & $Y$ & $Y$ \\
\hline - & - & - & - & - & - & - & - & - & - & - & - & - & - & - & - & $Y$ \\
\hline - & - & - & - & - & - & - & - & - & - & - & - & - & - & - & $Y$ & $Y$ \\
\hline - & - & $Y$ & - & - & - & - & - & - & - & - & - & - & - & - & $Y$ & $Y$ \\
\hline$Y$ & $Y$ & - & - & - & - & - & - & - & - & - & - & - & - & - & $Y$ & $Y$ \\
\hline - & - & - & - & - & - & - & - & - & - & - & $Y$ & $Y$ & - & - & - & - \\
\hline$Y$ & $Y$ & - & - & - & - & - & - & - & - & - & $Y$ & - & - & - & - & - \\
\hline- & - & - & - & - & - & - & $Y$ & $Y$ & - & - & $Y$ & - & - & - & - & - \\
\hline - & - & - & - & - & - & - & $Y$ & $Y$ & - & - & - & - & - & - & $Y$ & - \\
\hline - & - & $Y$ & - & - & - & - & $Y$ & $Y$ & - & - & - & - & - & - & $Y$ & - \\
\hline - & - & $Y$ & - & - & - & - & $Y$ & $Y$ & - & - & - & - & - & - & - & - \\
\hline
\end{tabular}


Table 4 Distribution of genetic mutation for isoniazid and rifampicin resistance

\begin{tabular}{|c|c|c|c|c|}
\hline Countries & $\begin{array}{l}\text { katG (n \%), top } 3 \\
\text { common loci }\end{array}$ & $\begin{array}{l}\text { inhA (n \%), top } 3 \\
\text { common loci }\end{array}$ & $\begin{array}{l}\text { ahpC (n \%), top } 3 \\
\text { common loci }\end{array}$ & $\begin{array}{l}\text { rpoB (n \%), top } 3 \\
\text { common loci }\end{array}$ \\
\hline Thailand (the present study) in the & $20 / 34(58.8 \%)$ & $27 / 34(79.4 \%)$ & $13 / 34(38.2 \%)$ & $28 / 34(82.3 \%)$ \\
\hline north of Thailand & $315,343,312,340$ & $14,114,84$ & $75,76,10,12,20$ & $507,508,445$ \\
\hline Thailand $^{32}$ (in the middle of Thailand & $25 / 29(86.21 \%)$ & $7 / 25(24.0 \%)$ & NA & NA \\
\hline in 2007) & $315,463,308$ & $(-15),(-8), 21$ & & \\
\hline \multirow[t]{2}{*}{ Myanmar $^{33}$} & $31 / 33(72.1 \%)$ & $2 / 33(4.7 \%)$ & NA & $32 / 33(97.0 \%)$ \\
\hline & 315 & $(-15)$ & & $531,526,516$ \\
\hline \multirow[t]{2}{*}{ People's Republic of China ${ }^{34}$} & I78/242 (73.9\%) & $26 / 242(10.7 \%)$ & $22 / 242(9.1 \%)$ & $213 / 242(88.0 \%)$ \\
\hline & $315,295,299$ & $(15),(-8), 25$ & $(-10),(-6),(-12)$ & $531,526,516$ \\
\hline \multirow[t]{2}{*}{ Poland ${ }^{35}$} & $40 / 50(80 \%)$ & $8 / 50(16 \%)$ & $5 / 50(10 \%)$ & NA \\
\hline & $315,463,234$ & $(-15),(-8), 289$ & $48,54,57$ & \\
\hline \multirow[t]{2}{*}{ Switzerland ${ }^{36}$} & $101 / 154(65.6 \%)$ & $35 / 154(22.7 \%)$ & NA & NA \\
\hline & 315 & $(-15)$ & & \\
\hline \multirow[t]{2}{*}{ Argentina $^{37}$} & $43 / 7 \mathrm{I}(60.5 \%)$ & I6/7| (22.5\%) & NA & NA \\
\hline & 315 & $(-15)$ & & \\
\hline \multirow[t]{2}{*}{ South Africa ${ }^{38}$} & NA & I65/232 (7I\%) & NA & NA \\
\hline & & $(-15),(-8)$ & & \\
\hline \multirow[t]{2}{*}{ Morocco $^{39}$} & $15 / 16$ (93.7\%) & $1 / 16(6.2 \%)$ & NA & $2 \mathrm{I} / 26(80.8 \%)$ \\
\hline & 315 & $(-15)$ & & $531,316,513,526$ \\
\hline India, Moldova, Philippines, and & $268 / 316(84.8 \%)$ & $101 / 316(32.0 \%)$ & NA & NA \\
\hline South Africa ${ }^{40}$ & 315 & $(-15),(-8),(-17)$ & & \\
\hline
\end{tabular}

Abbreviation: NA, not available.

were different from other parts of Thailand and other countries. The distribution of these mutations in certain populations must be studied before developing specific mutation test panels for each area. These data and proposed mutation panels can be applied for further molecular M/XDR-TB diagnosis in the upper northern region of Thailand.

\section{Acknowledgments}

We would like to thank all the staff members in DPC10 as well as Nakornping Hospital, Chiang Rai Hospital, Lampang Hospital, Phayao Hospital, Chiang Kam Hospital, Lamphun Hospital, Phrae Hospital, and Nan Hospital for their cooperation. This study was supported by the National Research Council of Thailand and the Faculty of Medicine, Chiang Mai University, Chiang Mai, Thailand. The study was also partly supported by the Thailand National Research University (NRU) Fund.

\section{Author contributions}

All authors contributed toward data analysis, drafting and critically revising the paper and agree to be accountable for all aspects of the work.

\section{Disclosure}

All the authors report no conflicts of interest in this work.

\section{References}

1. World Health Organization. Global tuberculosis report 2016. WHO/ HTM/TB/2016.13 ed. France: World Health Organization; 2016.
2. World Health Organization. Companion handbook to the WHO guidelines for the programmatic management of drug-resistant tuberculosis. Geneva, Switzerland: WHO Document Production Services; 2014.

3. Ahmad S, Mokaddas E. Current status and future trends in the diagnosis and treatment of drug-susceptible and multidrug-resistant tuberculosis. J Infect Public Health. 2014;7(2):75-91.

4. Liang L, Wu Q, Gao L, et al. Factors contributing to the high prevalence of multidrug-resistant tuberculosis: a study from China. Thorax. 2012; 67(7):632-638.

5. Lange C, Abubakar I, Alffenaar JW, et al; TBNET. Management of patients with multidrug-resistant/extensively drug-resistant tuberculosis in Europe: a TBNET consensus statement. Eur Respir J. 2014;44(1): 23-63.

6. Porwal C, Kaushik A, Makkar N, et al. Incidence and risk factors for extensively drug-resistant tuberculosis in Delhi region. PLoS One. 2013; 8(2):e55299.

7. Paramasivan CN, Lee E, Kao K, et al. Experience establishing tuberculosis laboratory capacity in a developing country setting. Int J Tuberc Lung Dis. 2010;14(1):59-64.

8. Parsons LM, Somoskövi A, Gutierrez C, et al. Laboratory diagnosis of tuberculosis in resource-poor countries: challenges and opportunities. Clin Microbiol Rev. 2011;24(2):314-350.

9. Boehme CC, Nabeta P, Hillemann D, et al. Rapid molecular detection of tuberculosis and rifampin resistance. $N$ Engl J Med. 2010;363(11): 1005-1015.

10. World Health Organization. Guidelines for the programmatic management of drug-resistant tuberculosis - 2011 update. Version March 2010. Available from: http://apps.who.int/iris/bitstr eam/10665/44597/1/9789241501583_eng.pdf. Accessed May 17, 2017.

11. Heysell SK, Houpt ER. The future of molecular diagnostics for drugresistant tuberculosis. Expert Rev Mol Diagn. 2012;12(4):395-405.

12. Lin SY, Desmond EP. Molecular diagnosis of tuberculosis and drug resistance. Clin Lab Med. 2014;34(2):297-314.

13. Chan RC, Hui M, Chan EW, et al. Genetic and phenotypic characterization of drug-resistant Mycobacterium tuberculosis isolates in Hong Kong. J Antimicrob Chemother. 2007;59(5):866-873.

14. Hofmann-Thiel S, van Ingen J, Feldmann K, et al. Mechanisms of heteroresistance to isoniazid and rifampin of Mycobacterium tuberculosis in Tashkent, Uzbekistan. Eur Respir J. 2009;33(2):368-374. 
15. Ilina EN, Shitikov EA, Ikryannikova LN, et al. Comparative genomic analysis of Mycobacterium tuberculosis drug resistant strains from Russia. PLoS One. 2013;8(2):e56577.

16. Lopez-Alvarez R, Badillo-Lopez C, Cerna-Cortes JF, et al. First insights into the genetic diversity of Mycobacterium tuberculosis isolates from HIV-infected Mexican patients and mutations causing multidrug resistance. BMC Microbiol. 2010;10:82.

17. Sherman DR, Mdluli K, Hickey MJ, et al. Compensatory ahpC gene expression in isoniazid-resistant Mycobacterium tuberculosis. Science. 1996;272(5268):1641-1643.

18. Almeida Da Silva PE, Palomino JC. Molecular basis and mechanisms of drug resistance in Mycobacterium tuberculosis: classical and new drugs. J Antimicrob Chemother. 2011;66(7):1417-1430.

19. Seifert M, Catanzaro D, Catanzaro A, Rodwell TC. Genetic mutations associated with isoniazid resistance in Mycobacterium tuberculosis: a systematic review. PLoS One. 2015;10(3):e0119628.

20. Miyata M, Pavan FR, Sato DN, et al. Drug resistance in Mycobacterium tuberculosis clinical isolates from Brazil: phenotypic and genotypic methods. Biomed Pharmacother. 2011;65(6):456-459.

21. Ameeruddin NU, Luke Elizabeth $\mathrm{H}$. Impact of isoniazid resistance on virulence of global and south Indian clinical isolates of Mycobacterium tuberculosis. Tuberculosis (Edinb). 2014;94(6):557-563.

22. Banerjee A, Dubnau E, Quemard A, et al. inhA, a gene encoding a target for isoniazid and ethionamide in Mycobacterium tuberculosis. Science. 1994;263(5144):227-230.

23. Li QJ, Jiao WW, Yin QQ, et al. Compensatory mutations of rifampin resistance are associated with transmission of multidrug-resistant Mycobacterium tuberculosis Beijing genotype strains in China. Antimicrob Agents Chemother. 2016;60(5):2807-2812.

24. Meftahi N, Namouchi A, Mhenni B, Brandis G, Hughes D, Mardassi H. Evidence for the critical role of a secondary site rpoB mutation in the compensatory evolution and successful transmission of an MDR tuberculosis outbreak strain. J Antimicrob Chemother. 2016;71(2): 324-332.

25. Pavankumar AR, Engström A, Liu J, Herthnek D, Nilsson M. Proficient detection of multi-drug-resistant Mycobacterium tuberculosis by padlock probes and lateral flow nucleic acid biosensors. Anal Chem. 2016;88(8):4277-4284.

26. Herrera L, Jiménez S, Valverde A, García-Aranda MA, Sáez-Nieto JA. Molecular analysis of rifampicin-resistant Mycobacterium tuberculosis isolated in Spain (1996-2001). Description of new mutations in the rpoB gene and review of the literature. Int J Antimicrob Agents. 2003;21(5):403-408.

27. Palomino JC, Martin A. Drug resistance mechanisms in Mycobacterium tuberculosis. Antibiotics. 2014;3(3):317-340.
28. World Health Organization. Guidelines for drug susceptibility testing for second-line anti-tuberculosis drugs for DOTS-plus 2001. Geneva: World Health Organization; 2001. Available from www.WHO_CDS_ TB_2001.288_eng.pdf. Accessed May 8, 2017.

29. Department of Disease Control, Ministry of Public Health, Bangkok, Thailand. [The Thailand's 2008 National MDR-TB Management Guidelines Bangkok, Thailand]: The Bureau of Tuberculosis of Thailand; 2001. Thai.

30. Afanas'ev MV, Ikryannikova LN, Il'ina EN, et al. Molecular characteristics of rifampicin- and isoniazid-resistant Mycobacterium tuberculosis isolates from the Russian Federation. J Antimicrob Chemother. 2007; 59(6):1057-1064

31. Valvatne H, Syre H, Kross M, et al. Isoniazid and rifampicin resistanceassociated mutations in Mycobacterium tuberculosis isolates from Yangon, Myanmar: implications for rapid molecular testing. J Antimicrob Chemother. 2009;64(4):694-701.

32. Khadka DK, Eampokalap B, Panitchakorn J, Ramasoota P, Khusmith S. Multiple mutations in katG and inhA identified in Thai isoniazidresistant Mycobacterium tuberculosis isolates. Southeast Asian J Trop Med Public Health. 2007;38(2):376-382.

33. Aung WW, Ei PW, Nyunt WW, et al. Phenotypic and genotypic analysis of anti-tuberculosis drug resistance in Mycobacterium tuberculosis isolates in Myanmar. Ann Lab Med. 2015;35(5):494-499.

34. Luo T, Zhao M, Li X, et al. Selection of mutations to detect multidrugresistant Mycobacterium tuberculosis strains in Shanghai, China. Antimicrob Agents Chemother. 2010;54(3):1075-1081.

35. Jagielski T, Bakuła Z, Roeske K, et al. Detection of mutations associated with isoniazid resistance in multidrug-resistant Mycobacterium tuberculosis clinical isolates. J Antimicrob Chemother. 2014;69(9):2369-2375.

36. Fenner L, Egger M, Bodmer T, et al; Swiss HIV Cohort Study and the Swiss Molecular Epidemiology of Tuberculosis Study Group. Effect of mutation and genetic background on drug resistance in Mycobacterium tuberculosis. Antimicrob Agents Chemother. 2012;56(6):3047-3053.

37. Imperiale BR, Zumárraga MJ, Di Giulio AB, Cataldi AA, Morcillo NS. Molecular and phenotypic characterisation of Mycobacterium tuberculosis resistant to anti-tuberculosis drugs. Int J Tuberc Lung Dis. 2013; 17(8):1088-1093.

38. Niehaus AJ, Mlisana K, Gandhi NR, Mathema B, Brust JC. High prevalence of inhA promoter mutations among patients with drug-resistant tuberculosis in KwaZulu-Natal, South Africa. PLoS One. 2015;10(9):e0135003.

39. Zakham F, Chaoui I, Echchaoui AH, et al. Direct sequencing for rapid detection of multidrug resistant Mycobacterium tuberculosis strains in Morocco. Infect Drug Resist. 2013;6:207-213.

40. Torres JN, Paul LV, Rodwell TC, et al. Novel katG mutations causing isoniazid resistance in clinical M. tuberculosis isolates. Emerg Microbes Infect. 2015;4(7):e42. 


\section{Supplementary material}

Table SI Primers used for sequencing

\begin{tabular}{|c|c|c|c|c|c|}
\hline Gene & Primer & Nucleotide sequencing $\left(5^{\prime}-3^{\prime}\right)$ & Product size $(\mathrm{pb})$ & Temperature $\left({ }^{\circ} \mathrm{C}\right)$ & Reference \\
\hline \multirow[t]{4}{*}{ katG } & MtkatGf & ACCCGAGGCTGCTCCGCTGG & 168 & $94^{\circ} \mathrm{C}-20 \mathrm{~s}$ & Afanas'ev' \\
\hline & & & & $50^{\circ} \mathrm{C}-20$ s 70 cycles & \\
\hline & & & & $72^{\circ} \mathrm{C}-20 \mathrm{~s}$ & \\
\hline & MtkatGr & CAGCTCCCACTCGTAGCCGT & & & \\
\hline \multirow[t]{4}{*}{$\operatorname{inh} A$} & MtfabGf & GCCTCGCTGGCCCAGAAAGG & 320 & $94^{\circ} \mathrm{C}-20 \mathrm{~s}$ & Afanas'ev' \\
\hline & & & & $56^{\circ} \mathrm{C}-20$ s 70 cycles & \\
\hline & & & & $72^{\circ} \mathrm{C}-20 \mathrm{~s}$ & \\
\hline & MtfabGr & CTCCGGATCCACGGTGGGT & & & \\
\hline \multirow[t]{6}{*}{$\operatorname{ahpC}$} & $\operatorname{ahpCI~F~}$ & GCCTGGGTGTTCGTCACTGGT & 359 & $\begin{array}{l}95^{\circ} \mathrm{C}-40 \mathrm{~s} 15 \mathrm{~min} \\
\text { (start) }\end{array}$ & Valvatne $^{2}$ \\
\hline & & & & $94^{\circ} \mathrm{C}-40$ s 30 cycles & \\
\hline & & & & $57^{\circ} \mathrm{C}-40 \mathrm{~s} I \mathrm{~min}$ & \\
\hline & & & & $72^{\circ} \mathrm{C}-40 \mathrm{~s} 15 \mathrm{~min}$ & \\
\hline & & & & (final) & \\
\hline & ahpC2 R & CGCAACGTCGACTGGCTCATA & & & \\
\hline \multirow[t]{4}{*}{$r p o B$} & MtrpoBf & GAGGCGATCACCGCAGAC & 321 & $94^{\circ} \mathrm{C}-20 \mathrm{~s}$ & Afanas'ev' \\
\hline & & & & $59^{\circ} \mathrm{C}-20$ s 70 cycles & \\
\hline & & & & $72^{\circ} \mathrm{C}-20 \mathrm{~s}$ & \\
\hline & MtrpoBr & GGTACGGCGTTTCGATGAAC & & & \\
\hline
\end{tabular}

\section{References}

1. Afanas'ev MV, Ikryannikova LN, Il'ina EN, et al. Molecular characteristics of rifampicin- and isoniazid-resistant Mycobacterium tuberculosis isolates from the Russian Federation. J Antimicrob Chemother. 2007;59(6):1057-1064.

2. Valvatne $\mathrm{H}$, Syre $\mathrm{H}$, Kross M, et al. Isoniazid and rifampicin resistanceassociated mutations in Mycobacterium tuberculosis isolates from Yangon, Myanmar: implications for rapid molecular testing. J Antimicrob Chemother. 2009;64(4):694-701.
Infection and Drug Resistance

\section{Publish your work in this journal}

Infection and Drug Resistance is an international, peer-reviewed openaccess journal that focuses on the optimal treatment of infection (bacterial, fungal and viral) and the development and institution of preventive strategies to minimize the development and spread of resistance. The journal is specifically concerned with the epidemiology of antibiotic

\section{Dovepress}

resistance and the mechanisms of resistance development and diffusion in both hospitals and the community. The manuscript management system is completely online and includes a very quick and fair peerreview system, which is all easy to use. Visit http://www.dovepress.com/ testimonials.php to read real quotes from published authors. 